\title{
Search for positron quasibound states in the doubly excited region of the helium atom
}

\author{
J. R. Machacek, R. Boadle, S. J. Buckman, and J. P. Sullivan \\ ARC Centre for Antimatter-Matter Studies, Research School of Physics and Engineering, \\ Australian National University, Canberra 0200, Australia \\ (Received 17 September 2012; published 5 December 2012)
}

\begin{abstract}
Recent calculations by Bromley et al. [Phys. Rev. Lett. 109, 063201 (2012)] have indicated that the $\operatorname{He}\left(2 s^{2}{ }^{1} S^{e}\right)$ state will bind a positron. The $e^{+} \mathrm{He}\left(2 s^{2}{ }^{1} S^{e}\right)$ state has a binding energy of $0.447 \mathrm{eV}$ with respect to the $\mathrm{He}\left(2 s^{2}{ }^{1} S^{e}\right)$ state. Binding of a positron to the $\mathrm{He} 2 s^{2}$ state has been predicted to give rise to two resonances in positron scattering from ground-state helium: $e^{+} \mathrm{He}\left(2 s^{2}{ }^{1} S^{e}\right)$ at $57.3716 \mathrm{eV}$ and a ${ }^{2} P^{o}$ shape resonance just above the $\mathrm{He}\left(2 s^{2}{ }^{1} S^{e}\right)$ threshold at $57.8485 \mathrm{eV}$. To investigate these predicted resonances, low-energy positron scattering experiments were performed to measure the total and positronium cross sections in the doubly excited region of helium in this energy region. No experimental evidence has been found for these resonances, but upper bounds on the resonance strength were determined for both predicted resonances.
\end{abstract}

DOI: 10.1103/PhysRevA.86.064702

PACS number(s): $34.80 . \mathrm{Uv}$

Theoretical calculations have predicted that many different atoms are capable of binding a positron to both the ground and electronically excited states of both atoms and molecules. For atomic systems, these states have binding energies ranging from $13 \mathrm{meV}\left(e^{+} \mathrm{Na}\right)$ to $0.50 \mathrm{eV}\left(e^{+} \mathrm{Ca}\right)$, but are unstable to positron-electron annihilation and therefore have short lifetimes [1]. Large Feshbach resonances have been observed in annihilation cross sections corresponding to the formation of positron-molecule bound states, providing strong evidence that such states also exist for molecules [2]. However, experimental evidence of positron-atom bound states has proven to be evasive.

Previous studies of low-energy positron scattering from atoms or molecules have yielded no evidence of positron-atom bound states [3,4]. Many of these investigations have been performed with relatively low-resolution $(\Delta E \geqslant 0.5 \mathrm{eV})$ and lowintensity positron beams, with the result that narrow or weak features in the cross sections were likely to be undetectable. In more recent years, the energy resolution available for positron scattering experiments has been improved significantly [5], but a high-resolution ( $\Delta E \sim 25 \mathrm{meV}$ ) investigation of $\mathrm{H}_{2}, \mathrm{~N}_{2}, \mathrm{CO}$, and $\mathrm{Ar}$ [4] found no sign of predicted quasibound states, or resonances, in the total scattering cross section. Positron-atom bound states with higher binding energies are more suited to experimental study, but nearly all of the atoms with high predicted binding energies are solids at room temperature. For example, some of the most promising candidates for the experimental search for positron-atom binding are openshell transition metals (e.g., Fe, Co, Ni) due to their high dipole polarizabilities and moderate ionization potentials [6]. Since single scattering positron experiments must use gaseous targets, atoms such as these transition metals provide an experimental challenge compared to the bulk of previous positron scattering studies.

The current search was motivated by recent calculations performed for positron binding in the region of the helium doubly excited states [7]. In their paper, Bromley et al. note the similarities in energetics of the doubly excited state(s) of helium to the ground state of the $\mathrm{Mg}$ ion, suggesting the likelihood that these state(s) can bind a positron. They also point out the similarity between the dipole polarizability $\left(\alpha_{d}\right)$ of He $2 s^{2}\left(76.2 a_{o}{ }^{3}\right)$ and $\mathrm{Mg} 3 s^{2}\left(71.3 a_{o}{ }^{3}\right)$ states. Positron binding was found to be possible for model alkali-metal atoms with dipole polarizabilities greater than $23.5 a_{o}{ }^{3}[1,3]$. The current study explores the region about two of the predicted resonant positions: the bound state of $e^{+} \mathrm{He}\left(2 s^{2}{ }^{1} S^{e}\right)$ and the ${ }^{2} P^{o}$ shape resonance just above the $\operatorname{He}\left(2 s^{2}{ }^{1} S^{e}\right)$ state energy. We report on the measurement of total and positronium (Ps) formation cross sections in these regions.

The apparatus and techniques used in these measurements have been discussed elsewhere [8], so only a summary is provided here. A moderated beam of positrons, produced by a $\sim 25 \mathrm{mCi}^{22} \mathrm{Na}$ source, was guided electrostatically from a neon moderator into a Surko buffer-gas trap. Positrons are confined in the radial direction by magnetic fields of approximately $0.05 \mathrm{~T}$. These positrons were trapped and cooled to near room temperature in an electrostatic potential well using $\mathrm{N}_{2}$ and $\mathrm{CF}_{4}$ buffer gases. The trapped positron cloud was released with an energy spread (FWHM) of $\sim 60 \mathrm{meV}$ and a repetition rate of $\sim 70 \mathrm{~Hz}$. A retarding potential analyzer (RPA) was used to determine the absolute energy and energy spread of the positron beam. This technique provides an absolute energy calibration which is accurate to better than $\pm 25 \mathrm{meV}$, and this has been validated by measurements of the positronium formation threshold in positron scattering from the noble gases [9]. The positrons passed through a scattering cell containing helium gas $\left(\sim 1.6 \times 10^{-3}\right.$ mbar $)$. An RPA located after the scattering cell was used to measure the loss of parallel energy of the positrons after their transit through the gas cell. The intensity of the positron beam was measured with a microchannel plate (MCP). The energy distribution was used to determine the partial cross sections, as has been explained previously [10].

This Brief Report presents the measurements made of the total and positronium (Ps) cross sections for positron scattering from helium. The total cross section is found using the BeerLambert law,

$$
\sigma_{T}=-\frac{1}{n l} \ln \left(\frac{I_{t}}{I_{0}}\right),
$$

where $n$ is the gas number density, $l$ is the scattering path length or length of the cell, $I_{t}$ is the transmitted intensity, and $I_{0}$ is the full incident intensity. The determination of other partial cross sections has been explained in a previous publication [11] 


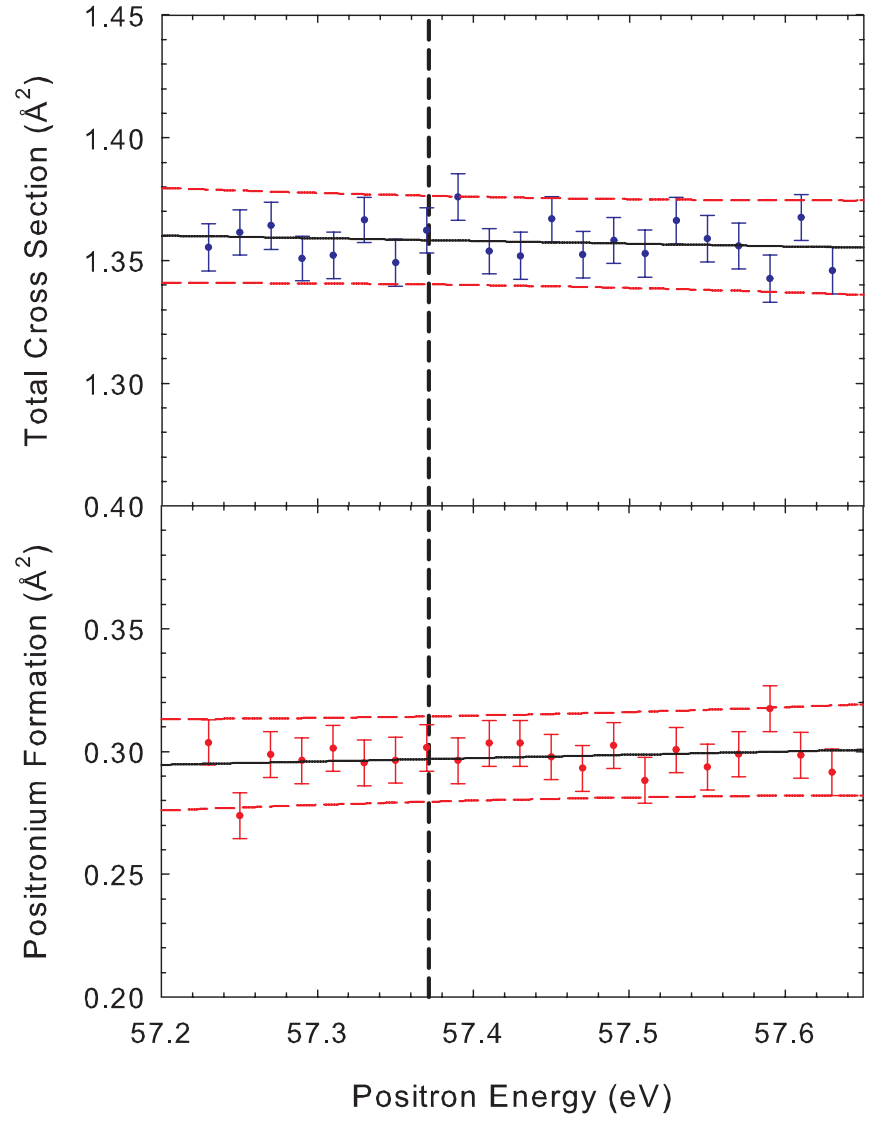

FIG. 1. (Color online) Cross sections: top panel, total scattering; bottom panel, positronium formation (Ps). The theoretically predicted location of the $e^{+} \mathrm{He}\left(2 s^{2}{ }^{1} S^{e}\right)$ state is indicated with a vertical dashed line at $57.3716 \mathrm{eV}$. The red dashed lines are the $95 \%$ confidence intervals and the solid lines are linear fits through the data.

Energy resolution and statistical uncertainties limit the sensitivity of these measurements to resonances in the cross sections. The upper bound on the resonance strength can be estimated (95\% confidence level) [4] by

$$
\sigma_{r} \Delta E_{r}<2 \sigma_{\text {rms }} \Delta E_{b},
$$

where $\sigma_{r}$ is the resonance cross section and $\Delta E_{r}$ is the resonance width. $\sigma_{\mathrm{rms}}$ is the statistical uncertainty (one standard deviation) on the cross-section data and $\Delta E_{b}$ is the energy resolution of the beam. Note that this is only strictly applicable for symmetric resonant profiles.

Measurements were made of the total and positronium cross sections at energies around the two predicted resonance positions: $57.3716 \mathrm{eV}$ [for $e^{+} \mathrm{He}\left(2 s^{2}{ }^{1} S^{e}\right)$ ] and just above the $\mathrm{He}\left(2 s^{2}{ }^{1} S^{e}\right)$ threshold of $57.8485 \mathrm{eV}$ (for the ${ }^{2} P^{o}$ shape resonance) [7]. The energy step size in the measurement was $20 \mathrm{meV}$. Figures 1 and 2 show the results for both energy regions. The statistical uncertainties in Fig. 1 range from $0.7 \%$ (total) to $3.1 \%$ (Ps), and in Fig. 2 from 1.3\% (total) to $6.4 \%(\mathrm{Ps})$. No resonant features are observed in these cross sections with this energy resolution and these statistical uncertainties.

Using (2), it is possible to place an upper bound on the resonance strength at each of these energies. For the $e^{+} \mathrm{He}\left(2 s^{2}{ }^{1} S^{e}\right)$ resonance, the upper bound is $0.95 \AA^{2} \mathrm{meV}$

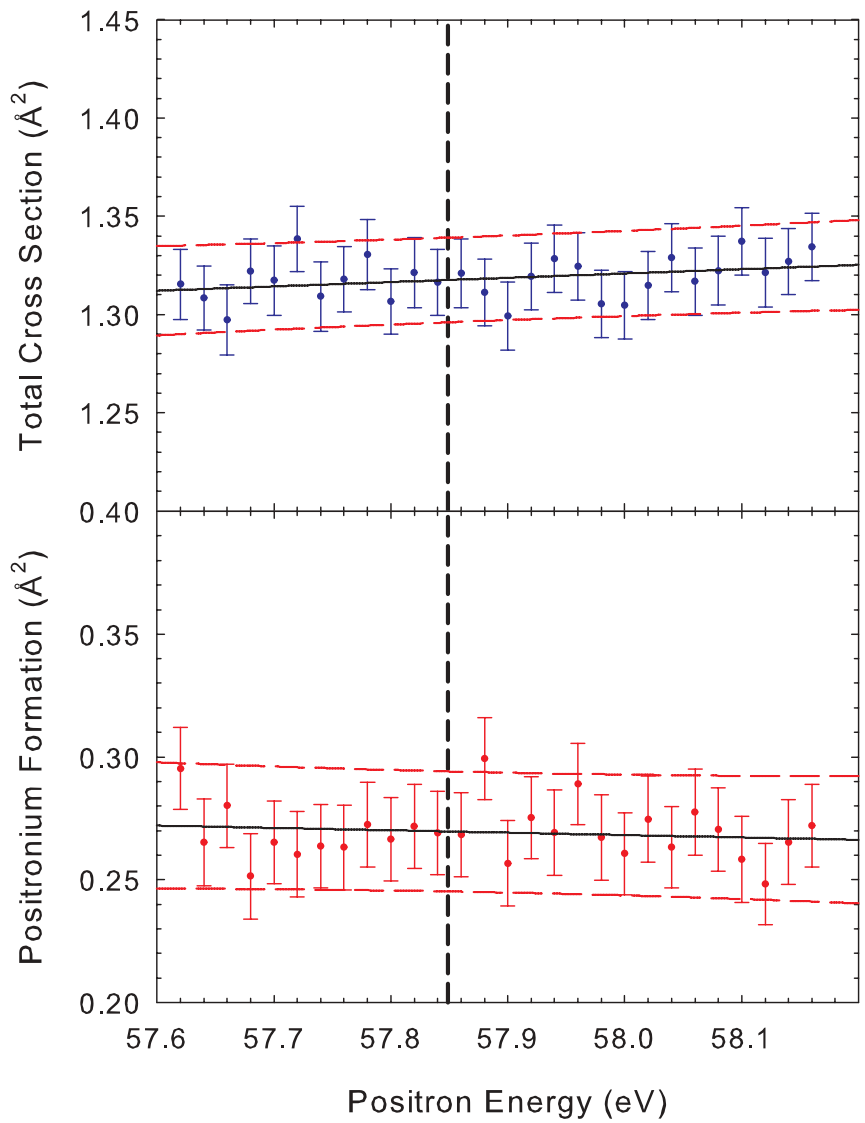

FIG. 2. (Color online) Cross sections: top panel, total scattering; bottom panel, positronium formation (Ps). The predicted theoretical location of the $\mathrm{He}\left(2 s^{2}{ }^{1} S^{e}\right)$ state at $57.8485 \mathrm{eV}$ is indicated with a vertical dashed line. The red dashed lines are the $95 \%$ confidence intervals and the solid lines are linear fits through the data.

for the total and $0.93 \AA^{2} \mathrm{meV}$ for the Ps cross section. For the ${ }^{2} P^{o}$ shape resonance, this is $1.72 \AA^{2} \mathrm{meV}$ for the total and $1.73 \AA^{2} \mathrm{meV}$ for the Ps cross section. These upper bounds are summarized in Table I.

Based on previous measurements of electron resonances in this energy region, Bromley et al. [7] compared positron attachment to the case of electron attachment, suggesting that these positron bound states are detectable. Using available experimental and theoretical results, we calculated the expected resonance strength. The magnitude of the observed electron resonance in the ion yield for the $\mathrm{He}\left(2 s^{2}{ }^{1} S^{e}\right)$ state was determined to be $0.8 \%$ of the total ion current by Quéméner et al. [12]. Using the results for ionization of helium by positron impact of Fromme et al. [13], who predict the magnitude

TABLE I. Summary of resonance searches.

\begin{tabular}{lcc}
\hline \hline $\begin{array}{l}\text { Energy } \\
\text { range }(\mathrm{eV})\end{array}$ & $\begin{array}{c}\text { Cross } \\
\text { section }\end{array}$ & $\begin{array}{c}\text { Upper bound on } \\
\text { resonance strength } \\
\left(\AA^{2} \mathrm{meV}\right)\end{array}$ \\
\hline $57.23-57.63$ & Total & 1.14 \\
$57.62-58.16$ & Ps & 1.12 \\
& Total & 2.06 \\
& Ps & 2.08 \\
\hline \hline
\end{tabular}


of the ionization cross section in this energy region to be $0.38 \AA^{2}$, we can determine an approximate resonance strength of $0.21 \AA^{2} \mathrm{meV}$ using Eq. (2). This resonance strength is more than five times smaller than our upper limit for measurements of the total and positronium formation cross sections about the $\mathrm{He}\left(2 s^{2}{ }^{1} S^{e}\right)$ state energy.

In conclusion, measurements of the total and positronium cross sections for $\mathrm{He}$ have been made in two different energy regions to search for resonances associated with the He $2 s^{2}$ state. While no clear resonances were observed, upper bounds have been placed on the strength of these resonances ranging from 1.12 to $2.08 \AA^{2} \mathrm{meV}$. This suggests that these resonances are either too narrow or too weak to detect with the current experimental arrangement and technique. It is possible that these resonances would be more pronounced in a different partial cross section (e.g., annihilation, direct ionization, or electronic excitation), warranting further study in the future as experimental techniques improve.

This work was supported by the Australian Research Council's (ARC) Centre of Excellence program. The authors would like to thank M. W. J. Bromley, J. Mitroy, and K. Varga for useful input and discussion of this work.
[1] J. Mitroy, M. W. J. Bromley, and G. G. Ryzhikh, J. Phys. B 35, R81 (2002).

[2] L. D. Barnes, S. J. Gilbert, and C. M. Surko, Phys. Rev. A 67, 032706 (2003).

[3] T. S. Stein, F. Laperriere, M. S. Dababneh, Y-F Hsieh, V. Pol, and W. E. Kauppila, 12th International Conference on the Physics of Electronic and Atomic Collisions (Gatlinburg, TN), edited by S. Datz (North-Holland Publishing company, Amsterdam, 1982), p. 424.

[4] J. P. Sullivan, S. J. Gilbert, S. J. Buckman, and C. M. Surko, J. Phys. B 34, L467 (2001).

[5] S. Gilbert, C. Kurz, R. Greaves, and C. M. Surko, App. Phys. Lett. 70, 1944 (1997).

[6] V. A. Dzuba, V. V. Flambaum, and G. F. Gribakin, Phys. Rev. Lett. 105, 203401 (2010).

[7] M. W. J. Bromley, J. Mitroy, and K. Varga, Phys. Rev. Lett. 109, 063201 (2012).
[8] J. P. Sullivan, A. Jones, P. Caradonna, C. Makochekanwa, and S. J. Buckman, Rev. Sci. Instrum. 79, 113105 (2008).

[9] A. C. L. Jones, P. Caradonna, C. Makochekanwa, D. S. Slaughter, R. P. McEachran, J. R. Machacek, J. P. Sullivan, and S. J. Buckman, Phys. Rev. Lett. 105, 073201 (2010).

[10] J. P. Sullivan, S. J. Gilbert, J. P. Marler, R. G. Greaves, S. J. Buckman, and C. M. Surko, Phys. Rev. A 66, 042708 (2002).

[11] A. C. L. Jones, C. Makochekanwa, P. Caradonna, D. S. Slaughter, J. R. Machacek, R. P. McEachran, J. P. Sullivan, S. J. Buckman, A. D. Stauffer, I. Bray, and D. V. Fursa, Phys. Rev. A 83, 032701 (2011).

[12] J. J. Quéméner, C. Paquet, and P. Marmet, Phys. Rev. A 4, 494 (1971).

[13] D. Fromme, G. Kruse, W. Raith, and G. Sinapius, Phys. Rev. Lett. 57, 3031 (1986). 